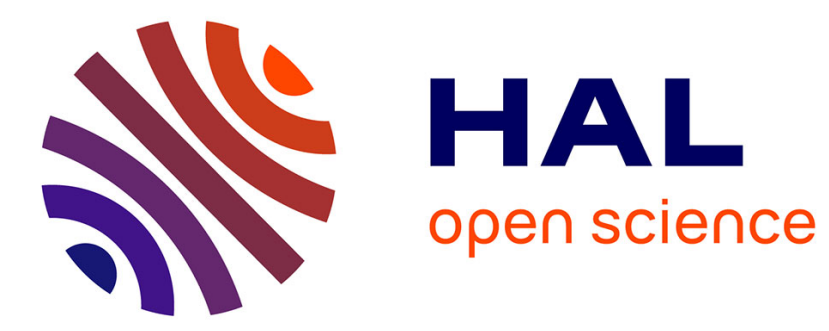

\title{
Les difficultés posées par les règles de compétence juridictionnelle et d'arbitrage de la CMR
}

\author{
Cécile Legros
}

\section{To cite this version:}

Cécile Legros. Les difficultés posées par les règles de compétence juridictionnelle et d'arbitrage de la CMR. Uniform law review, 2016, 21 (4), pp.577-590. 10.1093/ulr/unw033 . hal-02134015

HAL Id: hal-02134015

https://hal-normandie-univ.archives-ouvertes.fr/hal-02134015

Submitted on 20 May 2019

HAL is a multi-disciplinary open access archive for the deposit and dissemination of scientific research documents, whether they are published or not. The documents may come from teaching and research institutions in France or abroad, or from public or private research centers.
L'archive ouverte pluridisciplinaire HAL, est destinée au dépôt et à la diffusion de documents scientifiques de niveau recherche, publiés ou non, émanant des établissements d'enseignement et de recherche français ou étrangers, des laboratoires publics ou privés. 


\title{
Références de publication :
}

Les difficultés posées par les règles de compétence juridictionnelle et d'arbitrage de la CMR, Revue de Droit Uniforme/Uniform Law Review, Volume 21, Issue 4, Dec. 2016, pp. 577-590, https://doi.org/10.1093/ulr/unw033

\section{Les difficultés posées par les règles de compétence juridictionnelle et d'arbitrage de la CMR (droit français ; droit de l'Union européenne)}

\begin{abstract}
Certaines conventions internationales relatives au contrat de transport de marchandises comportent des règles spéciales de compétence. L'objectif de ces règles est de fournir un for le plus adapté possible à la spécificité de cette opération complexe qu'est l'opération de transport. À l'instar du droit commun et s'agissant de contrats internationaux, ces conventions prévoient souvent la possibilité pour les parties au contrat de choisir la juridiction compétente, voire de recourir à l'arbitrage. À défaut de choix, elles offrent des chefs de compétence qui diffèrent parfois de ceux issus du droit commun de la compétence internationale. C'est le cas de la convention internationale relative au transport de marchandises par route, dite CMR.

En principe compatibles avec le droit de I'Union européenne, voire avec le droit international pour ce qui est des règles relatives à l'arbitrage, les règles spéciales de compétence et d'arbitrage de la CMR posent toutefois quelques difficultés. La présente étude s'intéresse à trois types de difficultés assez mal connues : celle des options de compétence; celle de la spécificité des clauses attributives de juridiction ; et enfin, à l'encadrement strict des clauses d'arbitrage dans la CMR.
\end{abstract}

\section{Introduction}

La convention CMR est l'une des conventions internationales relatives au transport de marchandises qui comporte des règles relatives à la compétence juridictionnelle et à l'arbitrage, mais également à la reconnaissance et l'exécution des décisions judiciaires. C'est d'ailleurs la convention qui comporte les règles les plus détaillées sur ces questions. La CMR ne déroge pas à l'organisation générale des règles de compétence et d'arbitrage évoquées ci-dessus. L'article 31 de la CMR traite ainsi :

- de la compétence juridictionnelle (choix du juge compétent, règles de compétence à défaut de choix par les parties) en son paragraphe 1

- de la litispendance en son paragraphe 2

- du caractère exécutoire des jugements rendus en application de la convention en son paragraphe 3 (le paragraphe 4 définissant les jugements concernés)

- et enfin de l'interdiction de caution judicatum solvi en son paragraphe 5

S'agissant des règles de compétence juridictionnelle, et conformément aux règles généralement admises en matière de contrats internationaux, la CMR, comme plupart des conventions relatives au transport autorise ainsi le choix préalable par les parties dans le contrat de la juridiction qui sera compétente pour régler leurs différends ultérieurs. L'autonomie de la volonté se justifie ici par le caractère disponible des droits en jeu et ce, 
d'autant plus que ces contrats sont le plus souvent conclus entre professionnels. II s'agit cependant d'un choix qui est en général plus limité qu'en droit commun. L'article 31 offre subsidiairement au demandeur une option de compétence, traditionnelle en matière contractuelle, entre le "for du défendeur » et d'autres chefs de compétence. C'est dans cette dernière hypothèse que se manifeste la spécificité du droit des transports. Les fors offerts, qui sont plus nombreux qu'en droit commun, présentent en effet tous un lien objectif avec le contrat. Mais surtout, ils aboutissent à dispenser, dans la plupart des cas, les demandeurs d'aller plaider à l'étranger.

La jurisprudence rendue en application de cet article n'est pas très fournie. Pourtant l'étude des décisions des Etats partie à la convention auxquelles nous avons pu avoir accès, notamment via la base de données CMR UNIDROIT-IDIT ${ }^{1}$, montre là aussi certaines divergences de jurisprudence.

Nous ne pas traiterons pas ici des questions habituelles relatives au forum shopping, ou encore à l'articulation entre la CMR et les règles de droit européen, notamment en matière de litispendance ${ }^{2}$. Ces questions ont d'ailleurs été abordées par Simone Lamont-Black à propos des transporteurs successifs ${ }^{3}$. Trois difficultés, moins connues, ont retenu notre attention: celle des options de compétence; d'autres relatives aux clauses attributives de juridiction; et enfin, la spécificité de l'encadrement des clauses d'arbitrage dans la CMR.

Ces difficultés se doublent, dans I'Union Européenne, d'une considération supplémentaire qui est celle de l'articulation de ce texte avec les règles européennes ${ }^{4}$ relatives à la compétence, la reconnaissance et l'exécution des décisions étrangères (Règlement dit 'Bruxelles 1', et désormais 'Bruxelles 1 bis').

\section{Les difficultés posées par certaines options de compétence de l’article 31 \& 1 de la CMR}

$L^{\prime}$ article 31 paragraphe $1^{\text {er }}$ dispose que :

1. - Pour tous litiges auxquels donnent lieu les transports soumis à la présente Convention, le demandeur peut saisir, en dehors des juridictions des pays contractants désignées d'un commun accord par les parties, les juridictions du pays sur le territoire duquel :

a) le défendeur a sa résidence habituelle, son siège principal ou la succursale ou l'agence par l'intermédiaire de laquelle le contrat de transport a été conclu, ou :

b) le lieu de la prise en charge de la marchandise ou celui prévu pour la livraison est situé, et ne peut saisir que ces juridictions.

\footnotetext{
1 http://www.idit.asso.fr

${ }^{2}$ Case C 533/08, ECJ 4 May 2010, TNT Express Nederland [2010] ECR I 4107. Case C 452/12, ECJ 19 dec. 2013, Nipponka Insurance : C. LEGROS, "Incidence d'un jugement déclaratoire négatif de responsabilité rendu sur le fondement de la CMR sur la compétence du second juge saisi d'une action récursoire", JCP ed. E\&A (2014), 1480, comm. ${ }^{\circ} 12$. ; V. plus généralement : C. LEGROS, "Jurisdiction \& multimodal transport : a green perspective, MarIus, n459 (2015), 87-114. V. aussi : "Procédure d'insolvabilité et action en paiement du transporteur, quelle juridiction compétente ?" : C. LEGROS, Note sous CJUE 2 septembre 2014 - Nickel \& Goeldner Spedition / Kintra (Affaire C-157/13), Rev. crit. DIP (2015), 207-221.

${ }^{3}$ Cette revue, p.XX.

${ }^{4}$ Article 71 du Règlement $n^{\circ}$ 44/2001 concernant la compétence judiciaire, la reconnaissance et l'exécution des décisions en matière civile et commerciale; Article 71 du Règlement Bruxelles I bis, n $1215 / 2012$ du 12 décembre 2012 entré en application le 10 janvier 2015.
} 
Il ressort de cette disposition que le demandeur qui envisage d'intenter une action en justice relativement à un litige couvert par la convention dispose d'un choix entre différentes juridictions :

- une juridiction choisie (via une clause attributive de juridiction) ;

- une juridiction liée au domicile du défendeur (§a)

- différents fors optionnels précisés au § b : for du " défendeur »; for de " départ » de la marchandise; for "d'arrivée »; et enfin "for de conclusion » du contrat de transport.

C'est ce paragraphe $b$ qui constitue l'originalité du texte, car il prévoit deux fors originaux, du moins au regard du droit français : le for que j'appellerai " for de conclusion du contrat » (une règle comparable existe toutefois dans la jurisprudence française ${ }^{5}$ et dans le droit européen $\left.{ }^{6}\right)$. Et surtout le " for de départ " qui existe dans toutes les conventions relatives au contrat de transport de marchandises, parfois sous des formes différentes.

Il convient en outre de préciser que ce texte comporte une autre spécificité. En effet, l'article 31 ne désigne pas un juge compétent, mais prévoit la compétence " des juridictions d'un pays ". Le texte ne permet donc pas de connaître le juge spécialement compétent, mais seulement l'Etat dans lequel l'affaire pourra être jugée, ce qui constitue un premier point de difficulté. Se pose alors la question suivante : comment identifier, dans l'Etat désigné par la convention comme compétent, LA juridiction qui pourra statuer?

\section{1) Détermination de la juridiction spécialement compétente}

Si l'on se réfère au commentaire de la convention par Loewe, "La situation est au contraire que la CMR oblige les États contractants à mettre leurs juridictions à la disposition des parties dans les cas visés aux lettres a) et b), étant entendu que c'est au droit national de déterminer quel sera le tribunal compétent ratione materiae et ratione loci. " ${ }^{7}$. Ainsi, selon ce père fondateur de la $C M R$, le tribunal spécialement compétent doit être identifié par application de la lex fori, loi de l'Etat désigné par l'article 31. Ce renvoi à la lex fori pose certaines difficultés car il arrive que le droit du for ne contienne pas de règles permettant de déterminer le tribunal spécialement compétent. Tel semble le cas en droit néerlandais ${ }^{8}$. Ce renvoi est en revanche possible en droit français.

Prenons comme illustration un arrêt de la Cour d'appel de Bourges du 12 février $2002^{9}$. Selon cet arrêt : " L'article 31 de la CMR n'attribue nullement compétence automatique au tribunal du lieu de prise en charge ou à celui de la livraison. II renvoie en réalité aux règles de compétence des juridictions du pays sur le territoire duquel se trouve ce lieu de prise en charge ou de livraison. En application des articles 42 et 46 du CPC, la compétence retenue

\footnotetext{
${ }^{5}$ La jurisprudence dite « des gares principales » selon laquelle les personnes morales peuvent être assignées devant la juridiction dans le ressort de laquelle elles disposent d'une succursale ou d'une agence ayant le pouvoir de les représenter à l'égard des tiers a vocation à s'appliquer (T. com. Sète, 8 déc. 1964 : DMF (1966), 41 - CA Rouen, 18 févr. 1963: DMF (1966), 150 ; pour une application récente : CA Rouen, 2e ch., 24 avr. 2008 , JurisData $\mathrm{n}^{\circ} 2008-368842$, Rev. dr. transp. $\mathrm{N}^{\circ} 11$ (2008), n²39, p. 38, obs. M. NDENDE).

6 Art. $15-2^{\circ} \mathrm{RB}$ I ; Art. 7-5 ${ }^{\circ} \mathrm{RB}$ I bis.

${ }^{7}$ LOEwE R., Commentary on the Convention of 19 May 1956 on the Contrat for the International Carriage of Goods by Road (CMR), (1976) European Transport Law, 311.

8 Hoge Raad (Netherlands) (Summ.), 16 novembre 1990 Text: Nederlandse Jurisprudentie 1992/107 Rechtspraak van de Week 1990, 203.

${ }^{9}$ Cour d'appel de Bourges 12 février 2002 SAS Pluviaud Organisation c/. Sté De Klok Belgie N.V., CMR UNIDROIT-IDIT DataBase n${ }^{\circ} 12436$.
} 
doit être celle du lieu de livraison et non du lieu d'enlèvement des objets, ce qui attribue compétence aux juridictions belges, par ailleurs également compétentes au titre du lieu du domicile du défendeur ". La solution est constante comme l'illustre cette décision de la Cour d'appel de Reims du 25 mars 2014 ${ }^{10}$ : "L'article 31-1 de la CMR se bornant à attribuer une compétence générale aux juridictions du pays sur le territoire duquel est situé le lieu de la prise en charge de la marchandise ou le lieu prévu pour sa livraison sans toutefois désigner le tribunal compétent, il convient d'appliquer à titre supplétif les règles de compétence territoriale du pays où l'action doit être engagée, soit en l'occurrence les règles du droit français." ".

La Cour de cassation française n'a pas eu l'occasion d'énoncer ce principe de manière formelle, mais toutes les décisions qu'elle a rendues à propos de l'article 31 s'y conforment. En conséquence, les juridictions françaises font application des règles de procédure de droit commun, en l'occurrence celles du code de procédure civile français, à savoir : l'article 42 alinéa $1 \mathrm{du}$ Code de procédure civile qui pose le principe classique de la compétence du tribunal du domicile du défendeur ; l'article 43 définissant quant à lui la notion de domicile au regard des personnes physiques ou morales. En cas de pluralité de défendeurs, le demandeur saisit, à son choix en vertu de l'article 42, al. 2 du Code de procédure civile, la juridiction du lieu où demeure l'un d'eux ${ }^{11}$. Enfin, l'article 46 offre au demandeur une option de compétence en matière contractuelle : outre, le domicile du défendeur, le demandeur peut saisir la juridiction du lieu de la livraison effective de la chose ou du lieu de l'exécution de la prestation de service ${ }^{12}$. Le demandeur dispose donc d'une option supplémentaire entre d'une part, le lieu de livraison effective de la chose et, d'autre part, le lieu d'exécution de la prestation de service. Selon la jurisprudence, il s'agit du lieu "effectif", réel, et non du lieu "prévu" contractuellement ${ }^{13}$. Lorsque la livraison, ou l'exécution de la prestation se réalise en un lieu différent de celui prévu au contrat, le juge compétent n'est donc pas le même, ce qui crée tout de même une incertitude sur la compétence juridictionnelle, néfaste en matière contractuelle. De même, s'il n'y a pas de livraison effective ou pas d'exécution de la prestation, dans l'hypothèse par exemple ou la marchandise est perdue totalement en cours de transport, l'option de l'article 46 ne peut être utilisée de sorte qu'il n'est possible de saisir que le juge du défendeur ${ }^{14}$.

Ce renvoi à la lex fori pose toutefois certains problèmes. Le premier est celui de la rupture d'unité des solutions dans la mesure où les règles internes de compétence ne sont pas toutes identiques, voire parfois inexistantes. On peut d'ailleurs se demander si, lorsque le défendeur a sa résidence habituelle dans l'Union européenne, il ne conviendrait pas mieux d'appliquer les règles de compétence européennes. En effet, le droit de l'Union prévoit une

${ }^{10}$ CMR UNIDROIT-IDIT DataBase n41693; Bulletin des Transports et de la Logistique (2014) n³500, 231.

${ }^{11}$ Cass. com. 29 févr. 2000, N ${ }^{\circ} 95-17400$ : Bull. civ. 2000 IV n ${ }^{\circ} 41$ p. 35 ; Scapel (2000), n ${ }^{\circ}$, p. 10 ; Recueil Dalloz (2000), act. jurispr., 210 : au titre du for de la pluralité de défendeurs, l'assureur subrogé a valablement assigné le transitaire devant le tribunal du port de chargement dans le ressort duquel est établi le transporteur maritime.

12 T. com. Paris, 28 nov. 1979, DMF (1980), 422 ; Cass. $2^{\text {ème }}$ civ., 18 janv. 2001, n 96-20.912 : Bull. civ. 2001, II, $\mathrm{n}^{\circ} 10$, p. 6.

${ }^{13}$ Cass. com., 18 janv. 2001, ${ }^{\circ}$ 96-20912, Bull. civ. 2001, II n ${ }^{\circ} 10$ p. 6 : Le lieu de livraison effective de la chose en matière contractuelle s'entend, au sens de l'article 46 du nouveau Code de procédure civile, du lieu où la livraison a été ou doit être effectuée.

${ }^{14}$ Cour d'Appel de Nîmes, 13 déc. 1990, Sté Barral Cadière c/. Lauze : TRANSPORTS (1991), 623-638: En cas d'absence de livraison au destinataire, le seul lieu de livraison utile à la détermination de la juridiction compétente est celui de la remise au transporteur. 
articulation avec les conventions internationales comportant des règles de compétence. Il ne $s^{\prime}$ agit pas d'une exclusion réciproque de ces deux types de sources ${ }^{15}$.

La seconde difficulté résulte de ce que le renvoi à la loi nationale peut aboutir à des incohérences. Tel n'est pas le cas en droit français s'agissant de la compétence fondée sur le domicile du défendeur car ce chef de compétence existe dans notre droit interne (CPC, art. 42), comme d'ailleurs dans la plupart des systèmes juridiques. En revanche, des incompatibilités peuvent surgir s'agissant des fors optionnels. Au regard du droit français, c'est le for du lieu de prise en charge qui a posé le plus de difficultés.

\section{2) Contradiction entre le 'for de prise en charge de la marchandise' de l'article 31 \$1(b) et le droit français}

Aux termes de l'article 31 paragraphe 1, b) de la CMR, le demandeur peut saisir les juridictions de l'État sur lequel est situé "le lieu de la prise en charge de la marchandise ${ }^{16}$. Il s'agit de l'État où la marchandise a été confiée à la garde du transporteur. Le for du lieu de prise en charge est spécifique au contrat de transport. II se justifie par l'existence d'un lien objectif, matériel et juridique, avec le contrat de transport ${ }^{17}$. Si le dommage se réalise au 'départ' de la marchandise (au moment ou après le chargement), la compétence des juridictions localisées au lieu de prise en charge peut se justifier par la proximité. Ainsi les opérations d'expertise, la preuve, en seront facilitées. Mais l'exercice de l'option par le demandeur n'est pas conditionné par un lien objectif avec le litige. Le demandeur peut ainsi s'en prévaloir dans le cadre d'une action en responsabilité contre le transporteur alors même que le dommage a été constaté à destination. Ce for peut ainsi s'avérer plus contestable en ce qu'il consacre le forum actoris, pourtant traditionnellement écarté des instruments relatifs à la compétence ${ }^{18}$. Le domaine des transports est un domaine de professionnels où il n'est pas évident que le demandeur doive bénéficier d'une protection spéciale à l'instar des consommateurs ou des salariés dans les règlements européens.

En outre, la mise en œuvre de cette option par les juridictions françaises a posé certaines difficultés. En effet, dans la mesure où le for désigné par la convention est celui d'un Etat et non d'un juge précisément identifié, cette option ne peut en principe permettre de saisir le juge du lieu de prise en charge, que si le droit interne de l'Etat désigné par l'article 31 le permet. Or, comme nous l'avons vu précédemment, les articles 42 et 46 du Code de procédure civile ne permettent de saisir que le tribunal du lieu d'établissement du défendeur ou celui de la livraison effective de la marchandise. Dès lors, si un litige résultant d'un transport routier international au départ de la France peut en principe, aux termes de la CMR, être soumis aux juridictions françaises, en pratique, le droit français ne permet pas une telle saisine. II en est de même si la France est le lieu de destination en l'absence de livraison effective et alors que le transporteur est établi à l'étranger. Si les juges du fond ont parfois admis directement la compétence du lieu de prise en charge ${ }^{19}$, les hésitations étaient

\footnotetext{
15 voir infra.

${ }^{16}$ Les règles uniformes de la COTIF RU-CIM prévoient une disposition analogue La Convention de Hambourg également.

${ }^{17}$ Pour un exemple d'utilisation de ce for, V. CJCE, 28 oct. 2004, aff. C-148/03 : Recueil Dalloz (2005), jurispr. p. 547, note C. BRIERE ; Recueil Dalloz (2005), panorama de DIP, ${ }^{\circ}$ 15, p. 1263, obs. H. ChANTELOUP. - Chron. de droit des transports : JCP E 2005, I, 1360, p. 1928, obs. C. LEGROS.

18 V. notamment l'article 11 du préambule du RB I.

19 Cour d'appel de Rouen 12 janvier 1984, SA Marque Transports /. Sté Eurohaul, SA Jean, Thiers, SARL Guedj "Les Vignères", CMR UNIDROIT-IDIT DB n¹9858.
} 
permises $^{20}$. Ainsi, à suivre Lowe au sujet de la mise en œuvre des règles de compétence de la CMR : " II n'était pas dans l'intention des auteurs de permettre la saisine des tribunaux cités aux lettres a) et b) [de l'article 31, § 1] qu'à la condition que le droit national, en dehors de la CMR, prévoit lui-même leur compétence. ».

Pourtant, la Cour de cassation française a mis fin à ces hésitations par un arrêt de principe en 2002. Aux termes de l'arrêt Lutz rendu par la première chambre civile le 20 décembre $2000^{21}$. La Cour a ainsi écarté l'application stricte de la lex fori pour déterminer le tribunal spécialement compétent et admis la compétence de la juridiction du lieu de prise en charge de la marchandise au motif que : " en l'absence d'une règle de droit interne consacrant le critère de désignation du for prévu par l'article 31-1-b de la Convention de Genève du 19 mai 1956 "CMR", la juridiction saisie, qui ne peut refuser au demandeur le droit qu'il tient de ce texte de saisir une juridiction française, doit se reconnaître compétente dès lors qu'elle a un lien suffisant avec le litige ". Depuis cette décision, la jurisprudence est constante. Le plus souvent, il est reconnu que le lieu de prise en charge de la marchandise présente un lien suffisant avec le litige ${ }^{22}$. Plus rarement, d'autres lieux sont admis pour fonder la compétence du juge français.

La jurisprudence allemande adopte la même solution ${ }^{23}$. En l'absence de prise en charge de la marchandise par le transporteur, cette solution pose toutefois problème ${ }^{24}$. Reste que la position de la jurisprudence française ne semble pas respecter les principes d'interprétation de la Convention, du moins si l'on se fie au commentaire de Loewe à ce sujet ${ }^{25}$. Cette solution, empreinte d'un certain nationalisme, pourrait avoir pour seul objectif de reconnaître la compétence des juridictions françaises dès lors que l'un des plaideurs est français. Telle n'est cependant pas notre avis. Cette solution contribue en effet à corriger l'incohérence à laquelle aboutit l'application stricte des règles de la Convention. II est peu vraisemblable que les rédacteurs de la convention n'aient pas voulu assurer la compétence

\footnotetext{
${ }^{20}$ V. par ex. TC Lyon 12 février 1993, Sté Ravoux/Ste Europa SCA Express, BT 1993 p. 261, Note J. Tropin : «Le tribunal qui se fonde sur les dispositions de l'article 31 de la CMR pour retenir sa compétence et considère que c'est le tribunal du lieu de la prise en charge qui est compétent fait une interprétation erronée. C'est le pays de prise en charge qui est visé et la dévolution de compétence parmi les juridictions du pays choisi s'opère suivant les règles de droit interne, en l'espèce le domicile du défendeur ».

21 Cass. 1re civ., 20 déc. 2000, Stés Lutz et Sécuritas c/ Sté Frasgo et a., Bull. civ. I (2000) n 342, 221 ; CMR UNIDROIT-IDIT DB n 16269 ; Bulletin des Transports et de la Logistique (2001), 54-55 ; European Transport Law (2001), 237.

V aussi : Cass. com., 30 oct. 1984 : Bull. civ. 1984, IV, n 291 ; JCP (1985), IV, 14. - CA Rouen, 13 janv. 1998 : Bulletin des Transports et de la Logistique (1998), 361 ; Unif. L. Rev. / Rev. dr. Unif. (1998), 897. - CA Orléans, ch. com., 9 déc. 1999, Bulletin des Transports et de la Logistique (2000), n 2829-47, 12 ; Unif. L. Rev. / Rev. dr. Unif. (2000), 377. - CA Rouen, 30 mars 2006 : Bulletin des Transports et de la Logistique (2006), $\mathrm{n}^{\circ} 3126$, 263, qui déclare compétent le tribunal du lieu prise en charge de la marchandise en France, au motif qu'il est « conforme à une bonne administration de la justice »-Cass. com., 15 mai 2001 : Bulletin des Transports et de la Logistique (2001), n 2896-27.

22 Bordeaux, 2e ch., 20 mars 2007 : Bulletin des Transports et de la Logistique (2007) №3174, 37 ; Cass. com., 15 mai 2001, N98-18.603, Inédit ; Cass. com., 11 déc. 2001, № 00-10899, Bull. civ. IV (2001), n¹99, 194 ; Rouen, 2e chambre, 30 mars 2006 : Bulletin des Transports et de la Logistique (2006) n³126-24, 263 ; JCP (2006) $\mathrm{n}^{\circ} 26,2420$ (qui nie l'existence d'un lien suffisant du tribunal du domicile du transporteur et renvoie au tribunal du lieu de prise en charge) - Bordeaux, 2e ch., 20 mars 2007 : Bulletin des Transports et de la Logistique (2007), $\mathrm{n}^{\circ}$ 3174, 37 - TC Riom 9 décembre 2008: CMR UNIDROIT-IDIT DB n²3235; Bulletin des transports et de la Logistique (2009) $\mathrm{n}^{\circ}$ 3290, 612 - Paris Pôle 1 ch.1 24 juin 2010: CMR UNIDROIT-IDIT n²3365, Bulletin des Transports et de la Logistique (2010) $n^{\circ} 3329,483$.

23 Bundersgerichtshof, 13 mars 2014 : IZR 36/13, CMR UNIDROIT-IDIT DB n 41742.

${ }^{24}$ Handelsgericht Vienne, 03 avril 1984 : Transportrecht (1984), Fascicule 6, 152 ; CMR UNIDROIT-IDIT DB n ${ }^{\circ} 19857$.

${ }^{25}$ V. les observations de M. Tilche : Bulletin des Transports et de la Logistique (2007), $\mathrm{n}^{\circ} 3174,37$.
} 
d'un Etat désigné par l'article 31. Une telle question aurait pu faire l'objet d'un recours en interprétation devant la Cour internationale de justice de I'ONU tel que le prévoit la CMR en son article 47. La CIJ constitue en effet le seul véritable organe supra national d'unification ${ }^{26}$. Néanmoins, outre les freins de la lourdeur et du coût d'un recours, il ne semble pas que les juges nationaux aient connaissance de cette disposition. Une autre solution consisterait à appliquer systématiquement les principes d'interprétation des conventions internationales édictés par l'article 31 de la Convention de Vienne sur le droit des traités du 23 mai $1969^{27}$. Cependant, ces principes de droit international public ne sont pas familiers aux juges du fond des États partie à ces conventions. En particulier, en France, la convention de Vienne qui a une valeur coutumière faute d'avoir été ratifiée n'est quasiment jamais appliquée. Seule la communication internationale des décisions et la bonne volonté des juges peuvent contribuer à forger une jurisprudence autonome et uniforme ${ }^{28}$.

Le renvoi à la lex fori pour déterminer la compétence juridictionnelle est donc une source regrettable d'application non uniforme de la convention.

Une autre singularité de la CMR dans ce domaine réside dans le caractère non exclusif des clauses attributives de juridiction qu'elle autorise.

\section{L'originalité des clauses attributives de juridiction dans la CMR}

L'article 31 de la convention présente une autre originalité. S'il autorise le choix de la juridiction compétente par les parties, ce qui est du reste conforme aux principes de droit international privé en matière contractuelle, ce choix est cependant limité. D'une part les parties ne peuvent élire que le juge d'un Etat partie à la convention. D'autre part, les clauses d'electio juris ne sont pas exclusives, ce qui est plus contestable. Enfin, la convention ne précise pas la forme que doit revêtir le choix. La question de l'opposabilité de ces clauses est également passée sous silence.

\section{1) Choix exclusif des juridictions d'un Etat partie à la CMR}

Aux termes de l'article 31, les parties au contrat de transport routier international peuvent choisir la juridiction compétente pour régler leur différend ${ }^{29}$, à condition toutefois que la désignation porte sur un pays signataire de la Convention ${ }^{30}$. Cette condition se justifie par le souci d'assurer l'effectivité de l'application des dispositions substantielles de la Convention. Elle est ainsi considérée comme une limitation raisonnable de la liberté des parties ${ }^{31}$. Cependant, la convention n'impose pas l'existence d'un lien objectif entre le juge élu et le

\footnotetext{
${ }^{26}$ Voir nos propos introductifs, cette Revue, p.XX.

${ }^{27}$ W. Czapski, Application et interprétation de la convention CMR à la lumière du droit international, Unif. L. Rev. / Rev. dr. Unif. (2006), vol.XI, 545-568.

28 V. en ce sens Oberlandesgericht München (Allemagne), 23 juill. 1996 : Transportrecht (1997), 33 , 34 ; Unif. Law Rev. / Rev. dr. Unif. (1998), 207.

${ }^{29}$ Oberlandesgericht Hambourg (Allemagne), 26 avr. 1984 : Transportrecht (1984), Fasc. 7/8, 194.

${ }^{30}$ Oberlandsgericht Wien (Autriche), 15 oct. 1986 : Transportrecht, (1987), 223 : « La clause attributive de compétence déterminée par les parties ne peut attribuer la connaissance des litiges à une juridiction exclusive située hors du territoire du pays contractant dans la mesure où elles ne remplissent pas les conditions posées par l'article 31, § 1, a et b. », CMR UNIDROIT-IDIT DB n 19886.

${ }^{31}$ Ph. DelebecQue, Le nouveau droit international des transports in Mélanges J. Béguin, Litec, 2005, spéc. $\mathrm{n}^{\circ}$ 17 , p. 261, qui juge cette limitation raisonnable.
} 
contrat. Il semble donc possible d'élire un juge qui ne serait localisé ni dans l'État de départ de la marchandise, ni dans celui d'arrivée, ni même qui corresponde au lieu d'établissement de l'une des parties au contrat, pourvu que le juge élu soit celui d'un État partie à la convention.

La liberté des parties est donc assez grande, celles-ci pouvant même être mise à profit pour pratiquer le forum shopping, notamment en insérant des clauses attributives de juridiction donnant compétence à des fors qui connaissent les actions préventives en déclaration de non responsabilité, ou encore qui adoptent une conception restrictive de la faute équipollente au dol de l'article 29 de la convention. II ne semble pas toutefois que cette pratique soit très répandue.

\section{2) Absence d'exclusivité des clauses attributives de juridiction}

Les clauses attributives de juridiction de la CMR n'attribuent pas une compétence exclusive au juge élu, ce qui déroge aux principes contraires généralement reconnus en droit international privé. Le paragraphe 1er dispose ainsi que "le demandeur peut saisir, en dehors des juridictions des pays contractants désignées d'un commun accord par les parties, les juridictions du pays sur le territoire duquel ... ". La désignation d'un juge via une clause de compétence ne constitue ainsi qu'une option supplémentaire au profit du demandeur ${ }^{32}$. Une telle restriction est contestable car, dans la mesure où cette solution déroge au droit commun des clauses attributives de juridiction, elle est source d'insécurité juridique pour les parties qui risquent de s'y méprendre.

La jurisprudence française ne semble pourtant pas avoir été saisie de nombreux litiges à ce sujet. Plus fréquentes sont les décisions concernant la force obligatoire des clauses de compétence.

\section{3) Opposabilité des clauses attributives de juridiction}

Des clauses attributives de juridiction susceptibles de s'appliquer dans un litige relatif à un transport routier international peuvent être insérées dans différents types de documents. Dans la lettre de voiture elle-même, ce qui est assez rare en pratique. Ce type de stipulation peut toutefois être inséré dans la case $n^{\circ} 19$ de l'imprimé habituel des lettres de voiture CMR (« conditions particulières »). Plus fréquemment, ces clauses sont stipulées dans les conditions générales des opérateurs, transporteur ou commissionnaire. Dans ces hypothèses, se pose la question de savoir quelles parties à l'opération de transport sont liées par ces clauses.

Pour qu'une clause dérogatoire de compétence lie la partie qui ne l'a pas stipulée, il faut établir que celle-ci en a eu connaissance et y a consenti, au moment de la conclusion du contrat $^{33}$. A cet égard, la jurisprudence française fait une distinction entre l'opposabilité à l'égard de l'expéditeur ou du transporteur et l'opposabilité au destinataire.

\footnotetext{
32 contra: Oberlandesgericht Hambourg, 30 avril 1981, Transportrecht (1984), Fasc. 5, 132, qui admet que les parties ont pu convenir de la clause de compétence insérée aux conditions générales allemandes concernant le transporteur (ADsp) ; CMR UNIDROIT-IDIT DB n4 41256.

33 Cour d'appel d'Amiens 8 mars 2011 Sté Packard Bell BV et a./ SDV UK et autres, RG nº10/03357, CMR UNIDROIT-IDIT DB n²3481: «les documents comportant la référence aux Conditions générales de vente (BIFA) sont postérieurs à la formation du contrat de transport à l'origine du différend, et qu'il n'est pas démontré que l'expéditeur a eu connaissance et accepté lesdites conditions, la clause attributive de compétence ne peut lui être opposable. »
} 
Pr. Cécile Legros, Ph. D., Normandie Univ, UNIROUEN, CUREJ, 76000 Rouen, France

Directrice scientifique de l'Institut du droit international des transports (IDIT)

\section{- Opposabilité à l'expéditeur ou au transporteur}

S'agissant des clauses figurant dans les conditions générales du transporteur, la jurisprudence française se réfère en général, en l'absence de règle de la CMR sur ce point, à l'article 48 du CPC. Pourtant, si le juge désigné par la clause est un juge de l'Union, le droit européen devrait s'appliquer ${ }^{34}$.

Mais les décisions rendues en la matière sont très casuistiques. Parfois, la jurisprudence considère que " la seule mention des CGV contenant la clause attributive au verso des factures ne suffisant pas à caractériser l'acceptation du client, il n'y a pas lieu de lui opposer ladite clause ${ }^{35}$. D'autres fois, elle se montre moins exigeante ${ }^{36}$.

\section{- Opposabilité au destinataire}

S'agissant de l'opposabilité au destinataire, la Cour de cassation avait rendu un arrêt de principe en 2005, affirmant que " le consentement du destinataire au contrat de transport ne s'étend pas à la clause attributive de compétence qui, insérée dans la lettre de voiture, ne fait pas partie de l'économie du contrat et doit être acceptée par lui $"{ }^{37}$. Cette décision, exigeant l'acceptation spéciale du destinataire, relevait cependant du droit interne. II n'existe en revanche pas de décisions en matière de transport routier international. Mais il est sans doute possible de transposer ici les solutions du droit maritime. Pour ce qui est des clauses attributives de juridiction en droit européen, la ligne directrice est désormais posée par deux arrêts rendus en 2008 selon lesquels une clause attributive de juridiction convenue entre un transporteur et un donneur d'ordres et insérée dans un connaissement produit ses effets à l'égard d'un tiers (tel le destinataire) si ce dernier succède aux droits et obligations de l'expéditeur en vertu du droit national applicable. Dans le cas contraire, il convient de vérifier son consentement à la clause au regard des exigences de l'article 23 du règlement 44/2001 (et désormais de l'article 25 du règlement 1215/2012) ${ }^{38}$. En droit français, le destinataire ne succède pas aux droits de l'expéditeur. En effet, l'article $L$ 132-8 du code de commerce le considère comme une véritable partie au contrat de transport. Dès lors, la clause devrait le lier, ce qui n'est cependant pas la solution retenue en 2005 par la Cour de cassation. Si le "tiers » ne succède pas à l'expéditeur selon la loi applicable au contrat de transport, l'opposabilité de la clause relève alors de l'article 23 du Règlement 44/2001. La souplesse de ce texte en termes de consentement devrait ainsi conduire à déclarer ce type de clause plus facilement opposable au destinataire. II a ainsi été jugé que « Dès lors que le destinataire a expressément acquiescé par fax aux conditions générales transmises par fax également par le transporteur, conditions stipulant de façon très apparente une clause

\footnotetext{
34 Art. 23 du Règlement Bruxelles 1 ; art. 25 du Règlement Bruxelles 1 bis.

${ }^{35}$ Lyon, 3ème chambre, 11 mars 2011 Sté Kronospan / Sté Alloin Transorts : CMR UNIDROIT-IDIT DB n²3482; BTL n³359 (2011), 204 ; Rev. dr. Transp. (2011), 21 note I. BON-GARCIN.

36 Cour d'appel de Douai, 2ème ch., 10 septembre 2015, RG n 13/05015 : CMR UNIDROIT-IDIT DB n41752; BTL n³566 (2015), 542 : à propos d'une clause de compétence inscrite dans l'ordre de transport du commissionnaire déclarée opposable au transporteur.

${ }^{37}$ C. LEGROS, "Le destinataire de la marchandise : une partie "à part" du contrat de transport terrestre interne de marchandises", note sous Cass. com., 4 janvier 2005, JCP (2005), II.10067.

38 Cass. com. et Cass. civ. $1^{\text {ère }}, 16$ déc. 2008, Nº8-10460 et $\mathrm{N}^{\circ} 07-18834$, DMF (2009), nº 7000, note Ph. Delebecque.
} 
attributive de compétence au profit du tribunal de commerce de Salon de Provence, il ne peut invoquer l'exception d'incompétence au profit des juridictions de son pays ${ }^{39}$.

La jurisprudence maritime récente déclare notamment opposables des clauses insérées dans des connaissements, sur la base d'un usage existant entre les parties au contrat de transport ${ }^{40}$. Mais force est de constater qu'un tel usage n'existe pas dans le domaine du transport routier international.

\section{4) Forme des clauses attributives de juridiction}

La CMR ne se prononce pas sur la forme des clauses attributives de juridiction. Elle se contente d'exiger un accord entre les parties. Dès lors, cette question devrait être réglée selon les règles de droit international privé applicables à titre subsidiaire. Ainsi, à propos de la CMR, Loewe déclarait : "La CMR ne prévoit rien au sujet de la forme exigée d'un accord désignant une juridiction. L'appréciation de cette forme est ainsi laissée au droit national. On notera que la question de savoir si dans un tel contexte, il faut se référer au droit de l'État dont les parties ont prévu la juridiction ou au droit de l'accord des parties a été conclu est controversée. ". Ce renvoi au droit national est parfaitement contestable. En effet, pour le moins, le droit européen aurait vocation à s'appliquer si le juge élu est celui d'un Etat membre ${ }^{41}$. Pourtant, il est rare que les juridictions saisies de ces questions se réfèrent au droit européen, ce qui est, là aussi, une source de divergence de jurisprudence potentielle. La jurisprudence française a tendance, au mieux, à statuer sur le fondement de l'article 48 CPC. Le plus souvent les solutions, assez casuistiques, ne sont pas justifiées ${ }^{42}$. Une telle insécurité juridique n'est pas de nature à encourager le développement de ce type de clauses dans le transport routier international.

L'encadrement strict dont les clauses d'arbitrage font l'objet dans l'article 33 de la convention ne semble pas non plus propice au développement de ce mode de règlement des litiges dans le transport routier international.

\section{Les clauses d'arbitrage dans la CMR}

Le principe du recours à l'arbitrage est admis par différentes conventions relatives au contrat de transport international de marchandises. C'est le cas de la CMR.

L'article 33 dispose ainsi : " Le contrat de transport peut contenir une clause attribuant compétence à un tribunal arbitral à condition que cette clause prévoie que le tribunal arbitral appliquera la présente Convention». La formule " clause attribuant compétence à un tribunal arbitral " est maladroite car elle entretient une confusion entre pouvoir et compétence ${ }^{43}$. Elle crée en outre une ambiguïté avec les clauses attributives de juridiction.

\footnotetext{
39 Aix en Provence 0409 1996, Coene / Transports Gorlier, BTL (1997), 277 ; contra : Cour d'Appel de Gand (Belgique) 26 octobre 2001, Jurisprudence du Port d'Anvers (2003), 64-69.

40 Cass. com., 23 sept. 2014 , N 13-19108 et N¹3-21934, JDI (2015), 134-144, note C. LEGROS.

41 V. C. LEGRos, "Conflit de normes de matière de contrats de transport internationaux, Journal du Droit international" (2007), 3, 799 et 4, 1081.

42 Cour d'appel de Paris, 5e ch., 26 juin 1979, Biscuits Belin c/ Usitra-France et a. : « Ne constitue pas une attribution de compétence territoriale la clause figurant dans un article intitulé "Interprétation légale" et disposant que "le contrat sera applicable et interprété à tous égards conformément à la législation anglaise" ».

${ }^{43}$ V. notamment PH. THERY, Pouvoir et compétence, Thèse Paris II, 1974.
} 
Pr. Cécile Legros, Ph. D., Normandie Univ, UNIROUEN, CUREJ, 76000 Rouen, France

Directrice scientifique de l'Institut du droit international des transports (IDIT)

Si ce n'est cette maladresse de rédaction, l'article affirme bien le principe de la licéité du recours à l'arbitrage. Et contrairement aux clauses attributives de juridiction, il s'agit là d'une compétence exclusive ${ }^{44}$.

A la différences d'autres conventions transport, la CMR ne comporte qu'une seule exigence : que les arbitres appliquent la convention. Cette formule semble impliquer que la stipulation de l'application des règles de la convention CMR dans la clause est une condition de validité de la convention d'arbitrage. Telle est l'interprétation qui en est faite, tant par la jurisprudence française, qu'étrangère ${ }^{45}$, qui déclare nulles les conventions d'arbitrage ne se référant pas à la $C M R$, ou se référant à une loi étatique interne. Dans un arrêt du 27 juin 1979, la Cour d'appel de Paris a ainsi écarté une clause d'arbitrage qui prévoyait l'application de la loi suédoise ${ }^{46}$. La Cour d'appel d'Aix-en-Provence a accordé le même sort à une convention d'arbitrage faisant référence à la loi finlandaise ${ }^{47}$.

Cette jurisprudence nous semble critiquable même si elle correspond à la lettre et à l'esprit du texte car elle heurte les principes de l'arbitrage international. On peut en effet regretter une telle condition qui mélange le choix d'un mode de règlement des différends et celui de la loi applicable. La sanction de la nullité est sévère car elle méconnaît la volonté des parties de recourir à l'arbitrage. Certes, les arbitres à la différence des juges étatiques, ne sont pas contraints d'appliquer des règles impératives, ni même des conventions internationales impératives ${ }^{48}$. Comme l'a si bien exprimé Pierre Mayer, "l'arbitre n'a pas de for $"{ }^{49}$, de sorte que $s^{\prime}$ il applique une convention internationale ce n'est pas tant parce que ses conditions d'application sont réunies que parce qu'il estime ses règles appropriées au litige en jeu. Ainsi, les arbitres ne sauraient encourir le grief de dépassement de leur mission s'ils appliquent une convention impérative au détriment d'une loi choisie par les parties. II leur incombe cependant de rendre une sentence susceptible d'exécution et il n'est pas douteux que ces derniers appliqueraient la convention $C M R$, même si la clause se référait à une loi étatique interne. Pour conclure, nous estimons qu'une convention d'arbitrage ne renvoyant pas expressément à la CMR ne devrait pas être frappée d'emblée de nullité. La non application de la convention par les arbitres ne devrait être sanctionnée qu'au stade de l'exequatur, au titre de la méconnaissance de l'ordre public international ${ }^{50}$.

${ }^{44}$ OLG Koblenz (Allemagne) (Somm. angl.), CMR UNIDROIT-IDIT DB n41256; Transportrecht (2007), 249.

${ }^{45}$ Queen's Bench Division, commercial court (Royaume-Uni) $1^{\text {er }}$ décembre 1981 : CMR UNIDROIT-IDIT DB ${ }^{\circ} 19888$; Bofors UVA, CAC Ltd v. AB Skandia Transport (1982) Lloyd's Rep 410.

${ }^{46}$ BTL (1979), 440.

${ }^{47}$ Cour d'appel d'Aix-en-Provence, 2e ch. 2 septembre 2004 Cie Cigna et autres c/ Sté Daher, CMR UNIDROIT-IDIT DB n ${ }^{\circ} 21998$; BTL (2004), 614. ; Chronique de droit des transports, JCP E (2005), 1930, obs. C. LEGROS.

${ }^{48}$ Voir notamment l'article 1511 du CPC français (ancien article 1496) qui laisse un large pouvoir aux arbitres pour déterminer les règles applicables à défaut de choix par les parties.

${ }^{49}$ P. MAYER, "L'application par l'arbitre des conventions internationales", in L'internationalisation du droit : mélanges en l'honneur de Yvon Loussouarn, Paris, Dalloz, 1994, 275, spéc. n 4 et s. - P. Level, "Brèves réflexions sur l'office de l'arbitre", in Mélanges Perrot, p. 259, 265.

50 Article $1520 \mathrm{CPC}$; Article V\$2 de la Convention pour la reconnaissance et l'exécution des sentences arbitrales étrangères (New York, 1958).

La Convention pour la reconnaissance et l'exécution des sentences arbitrales étrangères signée à New York le 10 juin 1958 (la "Convention de New York »), est entrée en vigueur le 7 juin 195 
Pr. Cécile Legros, Ph. D., Normandie Univ, UNIROUEN, CUREJ, 76000 Rouen, France Directrice scientifique de l'Institut du droit international des transports (IDIT)

Mais il pourrait également être avancé que la stipulation d'une loi étatique d'un Etat partie à la convention CMR ne contrevient pas à la règle posée par l'article 33. En effet, dans les systèmes de droit international monistes, comme le système français, les conventions internationales régulièrement ratifiées font partie intégrante du droit français, de sorte que le choix de la loi française pour régir un contrat rend ipso facto ces conventions applicables.

Conclusion. Ces interrogations sur la compétence juridictionnelle ou les règles de la CMR relatives à l'arbitrage, du point de vue de la jurisprudence française, montrent bien que dans ce domaine aussi, des sources de divergence de jurisprudence existent. II conviendrait de remédier à ces divergences pour limiter le forum shopping. Cette pratique profite certes parfois à certaines parties bien conseillées par leurs avocats, mais elles tendent à ruiner à l'objectif d'uniformisation de la convention. L'encadrement des clauses d'arbitrage devrait également être assoupli si l'on souhaite développer ce mode de règlement des différents dans le domaine du transport routier international, ce qui n'est d'ailleurs peut-être pas opportun.

De manière générale, on peut constater que le caractère approfondi des règles des articles 31 et 33 est plutôt néfaste dans les rapports intra-européens, où ces règles se télescopent avec celles du droit européen qui suffiraient à régir la matière ${ }^{51}$. Peut-être conviendrait-il une fois pour toutes d'exclure ce type de règles dans les instruments internationaux lorsqu'un instrument de l'Union européenne est applicable. En revanche, dans les rapports avec des parties non européennes, à défaut de règles de compétence et d'arbitrage harmonisées au plan international, les règles de la $\mathrm{CMR}$, même si elles sont perfectibles, se révèlent à l'usage plutôt satisfaisantes.

51 C. LEGROS, "Jurisdiction \& multimodal transport : a green perspective", MarIus n459, 2015. 